Discussion/conclusion The central clinic achieved all BASHH targets, whereas the community clinics failed to do so, highlighting the need for a consistent approach to assessment of safeguarding concerns across all sites. Proforma completion is pivotal in safeguarding patients, however consistency between sexes is needed. Extending proforma use for reassessment and 16-17 year olds may also be beneficial. Improvements to ensure an equitable service are needed.

\section{P176 ARE SEXUALLY TRANSMITTED INFECTIONS ASSOCIATED WITH CHILD SEXUAL EXPLOITATION IN UNDER 16 YEAR OLDS ATTENDING GENITOURINARY MEDICINE CLINICS IN THE UK?}

${ }^{1}$ Chris Ward*, ${ }^{2}$ Gwenda Hughes, ${ }^{2}$ Holly Mitchell, ${ }^{3}$ Karen Rogstad. ${ }^{1}$ Central Manchester University Hospitals NHS Foundation Trust, Manchester, UK; ${ }^{2}$ Public Health England, London, UK; ${ }^{3}$ Sheffield Teaching Hospitals NHS Foundation Trust, Sheffield, UK

\subsection{6/sextrans-2016-052718.226}

Background Child sexual exploitation (CSE) is a challenging diagnosis to make, with few clinical signs or symptoms, and little evidence that markers such as sexually transmitted infections (STIs) are CSE predictors.

Aim To investigate associations between STIs and CSE risk factors.

Methods The genitourinary medicine clinic activity dataset (GUMCAD) was used to identify clinics with >18 STI diagnoses in 13-15 year-olds in 2012. Cases with confirmed bacterial or protozoal STIs were matched by age, gender and clinic with non-STI controls. Clinics provided details of CSE-related risk factors irrespective of STI presence through an on-line questionnaire. Associations between STI outcome and CSE-related risk factors were analysed using logistic regression.

Results 18/44 (40.9\%) clinics contacted provided data on 466 13-15 year-olds; 414 (88.8\%) were female, and 52 (11.2\%) male. $98.6 \%$ were heterosexual, and $66.7 \%$ white British. There were 18 (3.9\%) 13, 108 (23.2\%) 14 and 340 (80.0\%) 15 yearolds. In univariate analysis an STI diagnosis was significantly associated with: 'highly-likely' CSE (OR 9.00, $\mathrm{p}=0.037),>1$ partner (OR 5.50, $\mathrm{p}=0.000),>1$ attendance in $2012(\mathrm{OR}$ $3.79, \mathrm{p}=0.0000)$, safeguarding referral $(\mathrm{OR} 1.94, \mathrm{p}=0.022)$, other service involvement (OR 1.72, $\mathrm{p}=0.031$ ) and vulnerability (OR 1.64, $\mathrm{p}=0.026)$. After adjustment, STI diagnosis was significantly associated with: Health Advisor review (OR 6.78, $\mathrm{p}=0.000),>1$ partner (OR 5.82, $\mathrm{p}=0.002),>1$ attendance (OR 3.72, $\mathrm{p}=0.000$ ) and looked after child (OR 3.43, $\mathrm{p}=0.039$ ).

Discussion The presence of a bacterial or protozoal STI is only weakly associated with CSE and should not be used to infer CSE in the absence of more compelling evidence.

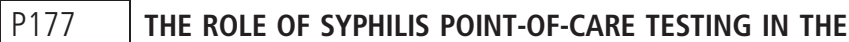 MANAGEMENT OF PATIENTS WITH GENITAL ULCERATION}

Rachel Hayward*, Meg Boothby. Whittall Street Clinic, Birmingham, UK

\subsection{6/sextrans-2016-052718.227}

Background/introduction Within the United Kingdom (UK) there has been an increase in infectious syphilis in the last decade, particularly amongst white men who have sex with men (MSM) aged 25-34 years old. Syphilis Point-of-Care (POC) tests were originally designed for resource-limited settings; however they can play a role in sexual health clinics in the UK.

Aim(s)/objectives Review the use of syphilis POC testing in the management of patients with genital ulceration.

Methods A search of Electronic Patient Records identified all patients who were offered a syphilis POC test between $1^{\text {st }}$ October 2014 and $31^{\text {st }}$ March 2015 at Whittall Street Clinic. Using a previously tested audit data collection tool, information about patient demographics, indication for syphilis POC test and clinical diagnosis were collected from each patient record and anonymised.

Results During six months, 111 records were identified as having offered a syphilis POC test. 13 records were excluded; 3 duplicate records and 10 patients were offered a syphilis POC test, but it was not performed. Of the remaining 98 records, 20 patients had a syphilis POC test performed due to genital ulceration. Eight patients had confirmed syphilis on serology testing, of which four had a reactive syphilis POC test on the day of presentation and subsequently had treatment the same day.

Discussion/conclusion Syphilis POC test remains an important diagnostic tool in settings which have no on-site laboratory facilities. Syphilis POC can also add strength to clinical judgement and diagnostic tests in well-resourced settings.

\section{P178 DOING IT THE FIRST TIME? AN AUDIT OF MEN REFUSING HIV TESTING AT FIRST CLINIC VISIT}

Emma Hardy*, Judith Russell, Sue Mitchell. Queen Elizabeth Hospital, Woolwich, UK

\subsection{6/sextrans-2016-052718.228}

Background/introduction South East London has the UK highest rates of heterosexual HIV acquisition and also significant numbers of HIV positive MSM. Our current uptake of HIV testing is $84 \%$. Risk assessment is the subject of the current BASHH national audit.

Aim(s)/objectives Trying to achieve highest possible uptake of HIV testing by GUM attenders we examined a group of new male attenders who refused HIV tests looking at their risk and reasons for refusal.

Methods A review of clinic notes of 50 consecutive male patients who refused an HIV test at first ever clinic visit comparing to the preceding new male patient accepting an HIV test.

Results

\begin{tabular}{|c|c|c|c|}
\hline & REFUSED $N=50$ & TESTED $N=50$ & \\
\hline MEDIAN AGE & 27 & 27 & \\
\hline MSM & $5(10 \%)$ & $7(14 \%)$ & NS \\
\hline GU SYMPTOMS & $23(46 \%)$ & $23(46 \%)$ & \\
\hline STI DIAGNOSED & $30(60 \%)$ & $13(26 \%)$ & P 0.001 \\
\hline NON-WHITE ETHNICITY & $11(22 \%)$ & $24(48 \%)$ & P 0.012 \\
\hline PREVIOUS HIV TEST & $22(44 \%)$ & $19(38 \%)$ & NS \\
\hline
\end{tabular}

Of 5 MSM refusing HIV test all had a reason documented 4 reporting recent test and 1 very recent exposure; only 21/45 $(47 \%)$ heterosexual men had documented reason for refusal. 